OC 8405 IDENTIFICATION OF AN MTB-SPECIFIC SOLUBLE HOST SIGNATURE FOR RISK OF DEVELOPMENT OF ACTIVE TB IN HIV-POSITIVE MTB-EXPOSED CONTACTS

${ }^{1}$ Joseph Mendy, ${ }^{2}$ Novel N Chegou, ${ }^{3}$ Harriet Mayanja-Kizza, ${ }^{2}$ Kim Stanley, ${ }^{4}$ Bonnie Thiel, ${ }^{5}$ Tom Ottenhoff, ${ }^{6}$ Stefan Kaufmann, ${ }^{4}$ Henry Boom, ${ }^{2}$ Gerhard Walzl, ${ }^{1}$ Jayne Sutherland* ${ }^{1}$ MRC Unit at London School of Health and Tropical Medicine, The Gambia; ${ }^{2}$ Stellenbosch University, South Africa; ${ }^{3}$ Makerere University, Kampala, Uganda; ${ }^{4}$ Case Western Reserve University, Cleveland, USA; ${ }^{5}$ Leiden University Medical Centre, The Netherlands; ${ }^{6}$ Max Planck Institute for Infection Biology, Berlin, Germany

\subsection{6/bmjgh-2019-EDC.10}

Background With 2 billion people infected with Mycobacterium tuberculosis (Mtb) worldwide, identification of those most atrisk of progressing to active disease would provide a targeted, cost-effective approach for preventative therapy strategies. The GC6-74 project recruited Mtb-exposed HIV-positive (HIV+) contacts from 5 African countries with the aim of identifying molecular and protein signatures for identification of 'at-risk' subjects by comparing those who progressed to active disease (progressors) to those who remained asymptomatic (controls).

Methods For this arm of the project, we analysed longitudinal samples from $12 \mathrm{HIV}$ + progressors and $28 \mathrm{HIV}$ +matched controls from Uganda (Makerere University, MAK) and South Africa (Stellenbosch University, SUN). Diluted whole blood was stimulated for 7 days with 7 Mtb-specific antigens plus controls. Supernatant was collected and a 38-plex multiplex assay performed following identification of confirmed progressors and controls.

Results The median time to progression to active disease was 510 days for SUN and 425 days for MAK participants. Baseline CD4 counts were 163 cells $/ \mu \mathrm{l}$ for progressors and $154 \mathrm{cells} / \mu \mathrm{l}$ for controls. Baseline responses showed significantly lower IL-4 production in progressors following ESAT-6/CFP-10 (EC) stimulation $(p=0.0309)$ and significantly higher macrophage-derived chemokine (MDC) following both Rv3019 and TB10.4 stimulation. For the time-point closest to progression, IL-10 production following EC stimulation and IFN- $\gamma$ production following Rv3019 stimulation were significantly higher in progressors than controls $(p=0.0024$ and $p=0.0028$ respectively). A combination of 12 analytes following EC and TB10.4 stimulation gave $84.4 \%$ and $91.1 \%$ correct classification respectively.

Conclusion We have defined a soluble signature for detecting likely progression to active TB in HIV + subjects 1 year prior to progression. Following validation in other cohorts, this could be exploited for development of a field-friendly test for targeted interventional therapy.

\section{OC 8415 A TRANSLATIONAL PRECLINICAL PLATFORM TO ASSESS THE CHEMOPROPHYLAXIS AND CHEMOPREVENTION DOSE-RELATIONSHIP OF MALARIA DRUGS: THE CASE STUDY OF M5717}

\footnotetext{
${ }^{1}$ Sofia Rebelo, ${ }^{1}$ Daniel Simão, ${ }^{1}$ Franscisca Arez, ${ }^{2}$ Diana Fontinha, ${ }^{2}$ Marta Machado, ${ }^{1}$ Tatiana Martins, ${ }^{3}$ Christoph Fischli, ${ }^{4}$ Claude Oeuvray, ${ }^{1}$ Manuel Carrondo, ${ }^{3}$ Matthias Rottman, ${ }^{4}$ Thomas Spangenberg, ${ }^{1}$ Catarina Brito*, ${ }^{4}$ Beatrice Greco, ${ }^{2}$ Miguel Prudencio, ${ }^{1}$ Paula M Alves. 'Instituto de Biologia Experimental e Tecnológica, Oeiras, Portugal; ${ }^{2}$ Instituto de Medicina Molecular, Lissbon, Portugal; ${ }^{3}$ Swiss Tropical and Public Health Institute, Basel, Switzerland; ${ }^{4}$ Merck Global Health Institute, Eysins, Switzerland
}

10.1136/bmjgh-2019-EDC.11

Major progress has been made in the control of malaria leading to significantly reduce the number of cases and deaths.
However, to reach the elimination stage, new tools will be needed, including combination of drugs capable of blocking the spread of malaria through chemoprophylaxis.

M5717 is a first-in-class compound that targets the Plasmodium Eukaryotic translation Elongation Factor 2, essential for protein synthesis. M5717 is highly potent against all developing stages of Plasmodium parasites and has a long half-life suggesting that a single dose development may be possible for cure, prophylaxis and transmission blocking activities. In vivo preclinical $\mathrm{PK} / \mathrm{PD}$ data indicates an increased exposure in the portal vein compared to peripheral circulation translating into a prophylactic activity at a lower dose than the curative one. M5717 is currently completing first-in-man studies with the objective of initiating clinical prophylactic development in 2019. Additional data to model the human prophylactic dose will be needed prior to initiate the studies to demonstrate clinical efficacy (phase 2).

We recently established a human cell-based platform for drug screening against Plasmodium liver-stage infection relying on human hepatic 3D cultures in bioreactors supporting rodent $P$. berghei infection with similar rates of infection and parasite development compared to existing models. The platform was validated by assessing the activity of currently used antimalarial drugs.

Here we report the data of a dose response experiment to establish the liver stage efficacious concentration of M5717 compared to atovaquone in Plasmodium-infected hepatic 3D cultures. The data obtained with this new model were confirmed using the in vivo model of $P$. berghei sporozoiteinduced infection in mice, demonstrating the validity of the Plasmodium-infected hepatic 3D cultures as an enabling technology for malaria drug development. These data are also providing additional evidence that M5717 should be developed as a chemoprophylactic agent for the prevention of malaria.

\section{OC 8431 CLINICAL RESEARCH AND SUSTAINABLE DEVELOPMENT IN SUB-SAHARAN AFRICA: THE IMPACT OF NORTH- SOUTH PARTNERSHIPS}

Agnes Saint-Raymond*, Martin Harvey, Maria Cavaller. European Medicines Agency, London, UK

\subsection{6/bmjgh-2019-EDC.12}

Background The European legislation introduced in 2004 (under article 58) a collaboration tool to increase access to high quality and effective medicines in low- and middleincome countries. The European Medicines Agency (EMA) can provide scientific opinions on medicines intended for significant public health needs, in partnership with the World Health Organisation (WHO) and the relevant 'target' non-EU regulatory authorities. This EU-Medicines4all (EU-M4all) initiative contributes to the broader Global Health Mandate of the EU.

Methods We contacted the pharmaceutical companies holding 'article 58' scientific opinions and compiled the number of actual approvals based on these opinions.

Results Nine medicines have been assessed so far, most of them for HIV/AIDS, tuberculosis, malaria and maternal/newborn health. Although this figure may appear low, the impact of the corresponding scientific opinions is much wider. Approvals were granted in 66 different countries worldwide, 38 of which are in Africa, based on these opinions. 